\title{
ON A GALOIS THEORY FOR INSEPARABLE FIELD EXTENSIONS
}

BY

JOHN N. MORDESON

\begin{abstract}
Heerema has developed a Galois theory for fields $L$ of characteristic $p \neq 0$ in which the Galois subfields $K$ are those for which $L / K$ is normal, modular and, for some nonnegative integer $e, K\left(L p^{e+1}\right) / K$ is separable. The related automorphism groups $G$ are subgroups of a particular group $A$ of automorphisms on $L[x] / x^{p^{e}+1} L[x]$ where $x$ is an indeterminate over $L$. For $H \subseteq G$ Galois subgroups of $A$, we give a necessary and sufficient condition for $H$ to be $G$-invariant. An extension of a result of the classical Galois theory is also given as is a necessary and sufficient condition for every intermediate field of $L / K$ to be Galois where $K$ is a Galois subfield of $L$.
\end{abstract}

Let $L$ be a field of characteristic $p \neq 0$. In [4], Heerema exhibits an automorphism group invariant field correspondence on $L$ which incorporates both the Krull infinite Galois theory and the purely inseparable, finite higher derivation theory [1]. The associated automorphism groups are subgroups of the group $A$ of all automorphisms $f$ of the local ring $L[\bar{x}]=L[x] / x^{p^{e}+1} L[x]$ such that $f(\bar{x})$ $=\bar{x}$ where $x$ is an indeterminate over $L, e$ is a nonnegative integer, $x p^{e}+1 L[x]$ is the ideal in $L[x]$ generated by $x^{p^{e}+1}$, and $\bar{x}$ is the coset $x+x^{p^{e}+1} L[x]$.

In this paper we determine further properties concerning this correspondence. We use the following notation: For $G$ a subgroup of $A, G_{L}=\{f \in G \mid f(L) \subseteq L\}$, $G_{0}=\{f \in G \mid f(a)-a \in \bar{x} L[\bar{x}]$ for all $a \in L\}$, and $L^{G}=\{a \in L \mid f(a)=a$ for all $f \in G\}$. For $K$ a subfield of $L, G^{K}=\{f \in G \mid f(a)=a$ for all $a \in K\}$.

For subgroups $H \subseteq G$ of $A$ which are Galois [4, Definition 3.6, p. 197], we give a necessary and sufficient condition (Theorem 1) for $H$ to be $G$-invariant [4, Definition 3.9, p. 198]. This result extends [4, Corollary 4.4, p. 200]. We then give a natural extension of a result of the classical Galois theory (Theorem 2), namely that if $H$ is $G$-invariant and $H_{0}=G_{0}$, then the quotient group $G / H$ is isomorphic to $G_{H}$ where $G_{H}$ is the group of all automorphisms of $L^{H}[\bar{x}]$ which are the identity on $L^{G}[\bar{x}]$.

For $K$ a subfield of $L$ such that $L / K$ is algebraic, we say that $L / K$ splits and write $L=S \otimes_{K} J$ if and only if $L$ is the field composite of $S$ and $J$ over $K$ where

Received by the editors December 3, 1974.

AMS (MOS) subject classifications (1970). Primary 12F15; Secondary $12 \mathrm{A55}$.

Key words and phrases. Higher derivations, normal field extension, modular field extension, purely inseparable field extension. Galois theory. 
$S$ is the maximal separable intermediate field of $L / K$ and $J$ is the maximal purely inseparable intermediate field of $L / K$. For $K$ Galois ([4, Definition 3.7, p. 197], $[4$, Theorem 3.1, p. 196]), we give some necessary and sufficient conditions for every Galois intermediate field of $L / K$ to split over $K$ (Theorem 3). This result is then applied to the invariance of $H_{0}$ in $G_{L}$ where $H \subseteq G$ are Galois subgroups of $A$ (Corollary to Theorem 3 ).

For a purely inseparable field extension $J / K$, we give a necessary and sufficient condition for $J / F$ to have a modular base for every intermediate field $F$ (Theorem 4). This result describes a necessary and sufficient condition for every intermediate field of $L / K$ to be Galois where $K$ is a Galois subfield of $L$ (Proposition 4).

For $K$ a subfield of $L$ such that $L / K$ is algebraic and $L / K$ splits, say $L=$ $S \otimes_{K} J$, the splitting is called nontrivial when $S \supset K$ and $J \supset K$ where $\supset$ denotes strict inclusion. If $F$ is an intermediate field of $L / K$, we use the notation $S F$ to denote the field composite of $S$ and $F$ over $S \cap F$. We often use the fact that a subfield $K$ of $L$ is Galois if and only if $L=S \otimes_{K} J$ where $S / K$ is normal and $J / K$ is modular with exponent $\leqslant e+1$ [4, Theorem 3.1, p. 196]. Definitions of modlar and modular base can be found in $[9$, p. 401] or [8, Definition 1.57, p. 53; Definition 1.21, p. 14]. If $J / K$ is a purely inseparable field extension of bounded exponent, then $J / K$ is modular if and only if $J / K$ has a modular base ([9, Theorem 1, p. 403] or [8, Proposition 1.56, p. 50]).

1. Group invariance. Let $H$ denote the group of all rank $p^{e}$ higher derivations on $L$ where the group operation on $H$ is defined in $[4$, p. 194]. We let $\Delta$ denote the isomorphism of $H$ onto $A_{0}$ defined in [4, Proposition 2.1, p. 194]. For $K$ a subfield of $L, H^{K}=\left\{d \in H \mid d_{i}(a)=0, i=1, \ldots, p^{e}\right.$, for all $\left.a \in K\right\}$ where we use the notation $d=\left\{d_{0}, d_{1}, \ldots, d_{p^{e}}\right\}$ for $d \in H$.

THEOREM 1. Let $H \subseteq G$ be Galois subgroups of $A$ and let $S$ denote the maximal separable intermediate field of $L / L^{G}$. Then $H$ is $G$-invariant if and only if either $L^{H} \subseteq S$ and $H_{L}$ is $G_{L}$-invariant, or $L^{H} \supseteq S, L^{H} / L^{G}$ splits, and $H_{0}$ is $G_{0}$-invariant.

Our proof of Theorem 1 uses the fact that there does not exist a purely inseparable modular field extension $J / K$ of bounded exponent with an intermediate field $F$ such that $J \supset F \supset K, J / F$ is modular, and for every modular base $M$ of $J / K$ every $m \in M$ has the same exponent over $F$ that it has over $K$. The following lemmas show that such a field extension does not exist. However we note in the following example that such a field extension exists if we drop the requirement that $J / F$ be modular.

EXAMPLE 1. Let $K=P(x, y, z), J=K\left(z^{p^{-2}}, z^{p^{-2}} x^{p^{-1}}+y^{p^{-2}}\right)$, and $F=$ 
$K\left(y^{p^{-1}}\right)$ where $P$ is a perfect field of characteristic $p \neq 0$ and $x, y, z$ are algebraically independent indeterminates over $P$. Then for every modular base $M$ of $J / K$ every $m \in M$ has the same exponent over $F$ that it has over $K$. Let $\left\{m_{1}, m_{2}\right\}$ be a modular base $J / K$. Then both $m_{1}$ and $m_{2}$ have exponent 2 over $K$. Suppose that $m_{2}$ has exponent 1 over $F$. Then $F=K\left(m_{2}^{p}\right)$. Thus $\left\{m_{1}, m_{2}\right\}$ is a modular base of $J / F$ contrary to the fact that $J / F$ is not modular.

LEMMA 1. Let $J / K$ be a field extension and $F$ an intermediate field of $J / K$. If $J / K$ and $J / F$ are modular, then $J / K\left(F \cap J^{p j}\right)$ is modular for $j=0,1, \ldots$

Proof. Let $j$ be a fixed nonnegative integer. Suppose $i$ is an integer such that $i \geqslant j$. Then $F \cap J p^{i}=F \cap J p^{i} \cap J p^{i} \subseteq K\left(F \cap J p^{j}\right) \cap J p^{i} \subseteq F \cap J p^{i}$. Thus $K\left(F \cap J p^{j}\right) \cap J p^{i}=F \cap J p^{i}$. Since also $F \supseteq K\left(F \cap J p^{j}\right)$ and $J / F$ is modular, $K\left(F \cap J p^{j}\right)$ and $J p^{i}$ are linearly disjoint over $F \cap J p^{i}$. Now suppose $i<j$. That $K\left(F \cap J p^{j}\right)$ and $J p^{i}$ are linearly disjoint over $\left(K \cap J^{p i}\right)\left(F \cap J p^{j}\right)$ follows from the following diagram, the modularity of $J / K$, and [5, Lemma, p. 162].

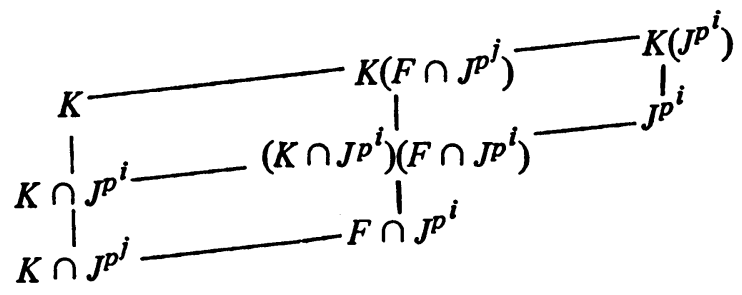

Q.E.D.

LEMMA 2. Let $J / K$ be a purely inseparable field extension with bounded exponent $n$ and let $F^{*}$ be an intermediate field of $J / K$ such that $F^{*} / K$ has exponent $\leqslant 1$. If $J / K$ and $J / F^{*}$ are modular and if for every modular base $M$ of $J / K$ every $m \in M$ has the same exponent over $F^{*}$ that it has over $K$, then $F^{*}=$ $K$.

Proof. Since $F^{*} / K$ has exponent $\leqslant 1, F^{*} \cap J p^{i} \subseteq K\left(K p^{-1} \cap J p^{i}\right)$ for $i=$ $0,1, \ldots, n$. There does not exist $a \in F^{*} \cap J p^{i}-K\left(K p^{-1} \cap J p^{i+1}\right)$ (set difference) else $a^{p^{-i}} \in K^{p^{-i-1}} \cap J-K^{p^{-i}}\left(K^{p^{-i-1}} \cap J^{p}\right)$ so $a^{p^{-i}} \in K^{p^{-i-1}} \cap J-$ $\left(K^{p^{-i}} \cap J\right)\left(K^{p^{-i-1}} \cap J^{p}\right)$. Thus $a^{p^{-i}}$ is in a modular base of $J / K$ [8, Proposition 1.55 (c), p. 49] and has exponent $i+1$ over $K$ and exponent $i$ over $F^{*}$, contrary to the hypothesis. Hence $F^{*} \cap J p^{i} \subseteq K\left(K^{p^{-1}} \cap J p^{i+1}\right), i=0,1, \ldots, n$. Since $J / K$ is modular, $K$ and $F^{*} \cap J p^{i}$ are linearly disjoint over $K \cap J p^{i}, i=0$, $1, \ldots$ Also since $J / F^{*}$ is modular, $F^{*}$ and $K\left(J p^{i+1}\right)$ are linearly disjoint over $K\left(F^{*} \cap J p^{i+1}\right), i=0,1, \ldots$, by [8, Lemma 1.60 (a), p. 55]. We have just seen that $F^{*} \cap J p^{i} \subseteq K\left(K^{p^{-1}} \cap J p^{i+1}\right) \subseteq K\left(J p^{i+1}\right)$ so $K\left(F^{*} \cap J p^{i}\right) \subseteq$ $K\left(J p^{i+1}\right), i=0,1, \ldots, n$. Since $K\left(F^{*} \cap \bar{J} p^{i+1}\right) \subseteq K\left(F^{*} \cap J p^{i}\right) \subseteq K\left(\overline{J p}^{i+1}\right)$, we have that $K\left(F^{*} \cap J p^{i}\right)=K\left(F^{*} \cap J p^{i+1}\right)$ for $i=0,1, \ldots, n$. Thus $F^{*}=$ 
$K\left(F^{*} \cap J^{p}\right)=\cdots=K\left(F^{*} \cap J p^{n}\right) \subseteq K$ so $F^{*}=K$. Q.E.D.

Lemma 3. Let $\mathrm{J} / \mathrm{K}$ be a purely inseparable field extension of bounded exponent $n$ and let $F$ be an intermediate field of $J / K$. If $J / K$ and $J / F$ are modular and if for every modular base $M$ of $J / K$ every $m \in M$ has the same exponent over $F$ that it has over $K$, then $F=K$.

Proof. Suppose $F \supset K$. Clearly every modular base of $J / K$ has the same property concerning exponents over any intermediate field of $F / K$. Since $F=$ $K\left(F \cap J p^{0}\right) \nsubseteq K$ and $K\left(F \cap J p^{n}\right) \subseteq K$, there exists a nonnegative integer $i$ such that $K\left(F \cap J p^{i}\right) \nsubseteq K$ and $K\left(F \cap J p^{i+1}\right) \subseteq K$. Set $F^{*}=K\left(F \cap J p^{i}\right)$. Then $F^{*} / K$ has exponent 1 and $J / F^{*}$ is modular by Lemma 1 . By Lemma $2, F^{*}=K$ which contradicts the assumption that $F \supset K$. Thus $F=K$. Q.E.D.

We also make use of the following lemma in the proof of Theorem 1.

Lemma 4. Suppose $F / K$ is an algebraic field extension such that $F=S \otimes_{K} J$ where $S$ is the maximal separable intermediate field and $J$ is the maximal purely inseparable intermediate field. Then the following conditions are equivalent:

(1) $F / K$ is modular.

(2) $F / S$ is modular.

(3) $J / K$ is modular.

PROOF. We first show that $\left(K \cap F p^{i}\right)\left(S p^{i}\right)=S \cap F^{p^{i}}, i=1,2, \ldots$ We have

$$
\begin{aligned}
K \cap F^{p^{i}} & =\left(K \otimes_{K p^{i}} 1\right) \cap\left(J p^{i} \otimes_{K p^{i}} S^{p^{i}}\right)=\left(K \cap J^{p^{i}}\right) \otimes_{K p^{i}} 1=K \cap J^{p^{i}}, \\
S \cap F^{p^{i}} & =\left(K \otimes_{K p^{i}} S^{p^{i}}\right) \cap\left(J^{p^{i}} \otimes_{K p^{i}} S^{p^{i}}\right) \\
& =\left(K \cap J^{p^{i}}\right) \otimes_{K^{p^{i}}} S^{p^{i}}=\left(K \cap J^{p^{i}}\right)\left(S^{p^{i}}\right) .
\end{aligned}
$$

Thus $\left(K \cap F p^{i}\right)\left(S p^{i}\right)=\left(K \cap J p^{i}\right)\left(S p^{i}\right)=S \cap F p^{i}, i=1,2, \ldots$ That (1) and (2) are equivalent is now apparent from the following diagram and [5, Lemma, p. 162].

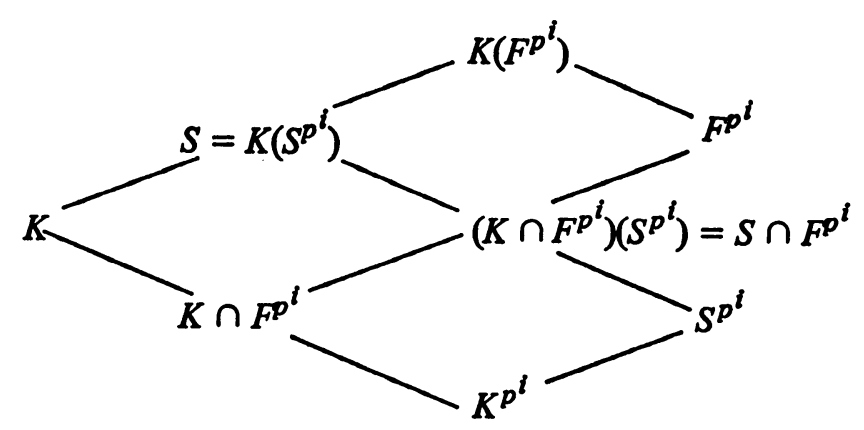

That (1) and (3) are equivalent follows from [8, Lemma 1.61(c), p. 56]. Q.E.D. Proof OF THEOREM 1. If $L / L^{G}$ is either separable or purely inseparable, 
then the theorem is trivially true. Hence suppose $L / L^{G}$ is inseparable but not purely inseparable. Let $J$ denote the maximal purely inseparable intermediate field of $L / L^{G}$. Assume $H$ is $G$-invariant. Then $L^{H} / L^{G}$ is normal by [4, Corollary 4.4, p. 200] so $L^{H} / L^{G}$ splits. Also $H_{0}$ is $G_{0}$-invariant and $H_{L}$ is $G_{L}$-invariant. Suppose $L^{H} \nsubseteq S$ and $L^{H} \nsupseteq S$. Since $L^{H} \nsubseteq S, L^{H} \cap J \supset L^{G}$. Since $H$ is Galois, $L^{H} J$ is modular over $L^{H}$ by $\left[4\right.$, Theorem 3.1, p. 196]. Thus $J /\left(L^{H} \cap J\right)$ is modular by Lemma 4. By Lemma 3, there exists a modular base $M$ of $J / L^{G}$ and an element $m$ of $M$ such that $m$ has exponent $n$ over $L^{G}$ and exponent $t$ over $L^{H} \cap$ $J$ with $n>t$. There exists a subset $X$ of $L^{G}$ such that $X \cup M$ is a $p$-base of $J$. Since $L / J$ is separable algebraic, $X \cup M$ is a $p$-base of $L$. Set $B=X \cup M$ and $C=\left\{b p^{i} \mid b \in B\right.$ and $i$ is the exponent of $b$ over $\left.L^{G}\right\}$. By [8, Proposition 1.22, p. 14], $C$ is a $p$-base of $L^{G}$. Since $L^{H} \nsupseteq S, S \supset L^{H} \cap S$. Let $s \in S-L^{H} \cap S$. Let $q$ be an integer such that $p^{e-n}<q \leqslant p^{e-n+1}$. Then there exists $d=$ $\left\{d_{0}, d_{1}, \ldots, d_{p}{ }\right\} \in H$ such that $d_{i}(m)=0, i=1, \ldots, q-1, d_{q}(m)=s$, and $d_{i}(b)=0\left(i=1, \ldots, p^{e}\right)$ for all $b \in B-\{m\}$. For all $c \in C-\left\{m p^{n}\right\}, d_{i}(c)=$ 0 for $i=1, \ldots, p^{e}$. Now $d_{i}\left(m p^{n}\right)=\left(d_{j}(m)\right)^{n}$ if $i=j p^{n}$ for some $j$ and $d_{i}\left(m p^{n}\right)$ $=0$ otherwise by $\left[10\right.$, p. 436]. Consider those $i$ such that $i=j p^{n}$. Then $1 \leqslant j$ $\leqslant p^{e-n}<q$ whence $d_{i}\left(m p^{n}\right)=0$. Thus $d \in H^{L} G$. Since $s \notin L^{H}$, there exists $h_{1} \in H_{L}$ such that $h_{1}(s)=s^{\prime} \in S$ with $s^{\prime} \neq s$. Now $p^{e-n+t}<q p^{t} \leqslant p^{e-n+t+1}$ $\leqslant p^{e}$ so $d_{q p}$ is defined. Also $m p^{t} \in L^{H} \cap J, m p^{t} \notin L^{G}$, and $d_{q p^{t}}\left(m^{p^{t}}\right)=$ $\left(d_{q}(m)\right) p^{t}=s^{p}$. For any integer $i$ such that $1 \leqslant i<q p^{t}$, we have that $d_{i}\left(m^{p^{t}}\right)$ $=\left(d_{j}(m)\right) p^{t}$ if $i=j p^{t}$ for some $j$ and $d_{i}\left(m p^{t}\right)=0$ otherwise. For those $i$ such that $i=j p^{t}, j p^{t}<q p^{t}$ so $j<q$. Thus $d_{i}\left(m p^{t}\right)=0$ when $1 \leqslant i<q p^{t}$. We now obtain a contradiction by showing that $H$ is not $G$-invariant. Thus either $L^{H} \subseteq S$ or $L^{H} \supseteq S$. We show that $H$ is not $G$-invariant by showing that $h_{1} g_{0}\left(m^{p^{t}}\right) \neq g_{0}\left(m^{p t}\right)$ where $g_{0}=\Delta(d)$. In the following we use the fact that $h_{1}$ is the identity on $J$. Now

$$
\begin{aligned}
h_{1} g_{0}\left(m p^{t}\right) & =h_{1}\left(m^{p^{t}}+\bar{x}^{q p^{t}} s^{p^{t}}+\sum_{i=q p^{t}+1}^{p^{e}} \vec{x}^{t} d_{i}\left(m^{p^{t}}\right)\right) \\
& =m^{p^{t}}+\bar{x}^{q p^{t}} s^{p^{t}}+\sum_{i=q p^{t}+1}^{p^{e}} \bar{x}^{i} h_{1} d_{i}\left(m p^{t}\right)
\end{aligned}
$$

Clearly $h_{1} g_{0}\left(m p^{t}\right) \neq g_{0}\left(m^{p t}\right)$ since $\left\{1, \bar{x}, \ldots, \bar{x}^{p^{e}}\right\}$ is linearly independent over $L$ and $s p^{t} \neq s^{\prime} p^{t}$.

Conversely, suppose $L^{H} \subseteq S$ and $H_{L}$ is $G_{L}$-invariant. Let $g \in G$ and $h \in H$. Then, by [4, Proposition 2.4, p. 195], $g=g_{1} g_{0}$ and $h=h_{1} h_{0}$ for unique $g_{1} \in G_{L}$, $g_{0} \in G_{0}, h_{1} \in H_{L}, h_{0} \in H_{0}$. Let $s \in L^{H}$. Since $g_{1}(s) \in S, g_{1}^{-1} h_{1} g_{1}(s) \in S$, and $g_{0}, h_{0}$ are identities on $S$, it follows easily that $g^{-1} h g(s)=s$. Thus $g^{-1} h g \in H$ so $H$ is 
$G$-invariant. Now suppose $L^{H} \supseteq S, L^{H} / L^{G}$ splits, and $H_{0}$ is $G_{0}$-invariant. Since $L^{H} \supseteq S$, we have that $H_{L}$ consists only of the identity map. Since $L^{H} / L^{G}$ splits, we have that $H_{0}$ is $G_{L}$-invariant by [4, Theorem 4.2, p. 199]. Thus if $g=g_{1} g_{0}$ $\in G$ and $h=h_{0} \in H=H_{0}$ where $g_{1} \in G_{L}$ and $g_{0} \in G_{0}$, we have $g^{-1} h g=$ $g_{0}^{-1} h_{0}^{\prime} g_{0}$ for some $h_{0}^{\prime} \in H_{0}$. Thus $g^{-1} h g \in H_{0}=H$ since $H_{0}$ is $G_{0}$-invariant. Hence $H$ is $G$-invariant. Q.E.D.

2. Quotient groups. Let $H \subseteq G$ be Galois subgroups of $A$. Let $G_{H}$ denote the group of all automorphisms $g_{H}$ for $L^{H}[\bar{x}]=L^{H}[x] / x^{p^{e}+1} L^{H}[x]$ such that $g_{H}(\bar{x})=\bar{x}$ and $g_{H}$ is the identity on $L^{G}$. If $H$ is $G$-invariant, then $H_{0}=G_{0}$ or $H_{0}=H$ by Theorem 1. In this section, $S$ denotes the maximal separable intermediate field of $L / L^{G}$ and $J$ denotes the maximal purely inseparable intermediate field of $L / L^{G}$.

THEOREM 2. Let $H \subseteq G$ be Galois subgroups of $A$. If $H$ is G-invariant and $H_{0}=G_{0}$, then $G / H \cong G_{H}$.

Proof. Define the mapping $\Phi$ on $G$ by, for all $g \in G, \Phi(g)$ is the restriction of $g$ to $L^{H}[\bar{x}]$. Since $H$ is $G$-invariant and $H_{0}=G_{0}, L^{G} \subseteq L^{H} \subseteq S$ and, $L^{H} / L^{G}$ is normal. Let $g=g_{1} g_{0} \in G$ where $g_{1} \in G_{1}, g_{0} \in G_{0}$. Since $L^{H} \subseteq S, g_{0}$ is the identity on $L^{H}[\bar{x}]$. Since $L^{H} / L^{G}$ is normal, $g_{1}\left(L^{H}\right)=L^{H}$. Thus $g\left(L^{H}\right)=L^{H}$ so $g\left(L^{H}[\bar{x}]\right)=L^{H}[\bar{x}]$. Hence $\Phi$ maps $G$ into $G_{H}$ and it follows easily that $\Phi$ is a homomorphism. Let $g_{H} \in G_{H}$. Since $L^{H} \subseteq S, g_{H}\left(L^{H}\right)=L^{H}$. Since $L^{H} / L^{G}$ is normal, the restriction of $g_{H}$ to $L^{H}$ can be extended to an automorphism of $S$. This extension can be extended to an element $g_{H}^{*}$ of $G$ by requiring it to be the identity on $J[\bar{x}]$. Thus for all $g_{H} \in G_{H}, g_{H}^{*} \in G$ and $\Phi\left(g_{H}^{*}\right)=g_{H}$. Hence $\Phi$ maps $G$ onto $G_{H}$. Since every element of $H$ is the identity on $L^{H}[\bar{x}], H \subseteq \operatorname{Ker} \Phi$. Since $H$ is Galois, $H=\left\{f \in A \mid f(a)=a\right.$ for all $\left.a \in L^{H}\right\}$. Thus since every $f \in \operatorname{Ker} \Phi$ is the identity on $L^{H}, f \in H$ so $\operatorname{Ker} \Phi \subseteq H$. Therefore $H=\operatorname{Ker} \Phi$ whence $G / H \cong G_{H}$. Q.E.D.

The following example shows that if $H \subseteq G$ are Galois subgroups of $A$ such that $H$ is $G$-invariant, then it is not necessarily the case that $L^{H}[\bar{x}]=\{q(\bar{x}) \in$ $L^{H}[\bar{x}] \mid h(q(\bar{x}))=q(\bar{x})$ for all $\left.h \in H\right\}$ even though $L^{H}=\{a \in L \mid h(a)=a$ for all $h \in H$.

Example 2. Let $L=P(u, v)$ and $K=P\left(u^{p}, v^{p}\right)$ where $P$ is a perfect field of characteristic $p \neq 0$ and $u, v$ are algebraically independent indeterminates over $P$. Let $H$ be the group of all rank $p^{e}$ higher derivations on $L$ with $e=0$. Set $G=\Delta\left(H^{K}\right)$ and $H=\Delta\left(H^{K(u)}\right)$. Let $d=\left\{d_{0}, d_{1}\right\} \in H^{K(u)}$ and set $h=\Delta(d)$. Then $h(u+\bar{x} v)=u+\bar{x} d_{1}(u)+\bar{x} v+\bar{x}^{2} d_{1}(v)=u+\bar{x} v$. However $u+\bar{x} v \notin$ $L^{H}[\bar{x}]=K(u)[\bar{x}]$. This example also shows that $H^{L^{H}}$ can be $H^{L}{ }^{G}$-invariant without $L^{H}$ being invariant under every $d \in H^{L} G$. Let $d=\left\{d_{0}, d_{1}\right\} \in H^{L} G$ be 
such that $d_{1}(u)=v$. Then $L^{H}$ is not invariant under $d$. However for all $d=$ $\left\{d_{0}, d_{1}\right\} \in H^{L} G$ and for all $d^{\prime}=\left\{d_{0}^{\prime}, d_{1}^{\prime}\right\} \in H^{L^{H}}, d^{-1} d^{\prime} d=\left\{d_{0}^{\prime},-d_{1}^{\prime}\right\} \in H^{L^{H}}$ so $H^{L}{ }^{H}$ is $H^{L}{ }^{G}$-invariant.

In view of Example 2 and [2, Corollary 3.6], the existence of a theorem corresponding to Theorem 2 for the case $H_{0}=H$ is unlikely. However we do have the following partial results.

Proposition 1. Let $H \subseteq G$ be Galois subgroups of $A$. If $H$ is $G$-invariant and $H_{0}=H$, then $G_{L} \cong\left(G_{H}\right)_{L} H$.

Proof. Define the mapping $\Phi$ on $G_{L}$ by, for all $g_{1} \in G_{L}, \Phi\left(g_{1}\right)$ is the restriction of $g_{1}$ to $L^{H}[\bar{x}]$. For $g_{1} \in G_{L}, g_{1}$ is the identity on $J$ and $g_{1}(S)=S$. Thus $g_{1}\left(L^{H}[\bar{x}]\right)=L^{H}[\bar{x}]$. Hence it is clear that $\Phi$ is a homomorphism of $G_{L}$ into $\left(G_{H}\right)_{L}$. Let $g_{H} \in\left(G_{H}\right)_{L} H$. Then $g_{H}$ is the identity on $L^{H} \cap J$ since $L^{H}=$ $S \otimes_{L} G\left(L^{H} \cap J\right)$. Now $g_{H}$ has a unique extension $g_{H}^{*}$ to an element of $G_{L}$, namely $g_{H}^{*}$ is the identity on $J[\bar{x}]$. The existence of the extension implies $\Phi$ maps $G_{L}$ onto $\left(G_{H}\right)_{L} H$ while the unicity of the extension implies that $\Phi$ is oneone. Q.E.D.

Let $G^{\prime}=\left\{g \in G \mid g\left(L^{H}[\bar{x}]\right)=L^{H}[\bar{x}]\right\}$ where $H \subseteq G$ are Galois subgroups of $A$. Then $G^{\prime}$ is a subgroup of $G$ and $H \subseteq G^{\prime}$.

Proposition 2. Let $H \subseteq G$ be Galois subgroups of $A$ such that $H$ is $G$ invariant and $H_{0}=H$. If $L=\bar{L}^{H} \otimes_{S} J^{\prime}$ for some intermediate field $J^{\prime}$ of $L / S$ such that $L^{H} / S$ and $J^{\prime} / S$ are modular, then $G^{\prime} / H \cong G_{H}$.

Proof. Define the mapping $\Phi$ on $G^{\prime}$ by for all $g^{\prime} \in G^{\prime}, \Phi\left(g^{\prime}\right)$ is the restriction of $g^{\prime}$ to $L^{H}[\bar{x}]$. Since $L=L^{H} \otimes_{S} J^{\prime}$ with $L^{H} / S$ and $J^{\prime} / S$ modular, every element in $G_{H}$ has an extension to an element of $G^{\prime}$ by [2, Theorem 3.4]. The remainder of the proof follows in an entirely similar manner to that of Theorem 2. Q.E.D.

3. Splitting. An exceptional field extension is one which is inseparable but has no elements (except those in the base field) which are purely inseparable over the base field ([3], [7]). A reliable field extension is one which is generated by every relative $p$-base [7].

We let $S$ denote the maximal separable intermediate field and $J$ the maximal purely inseparable intermediate field of the field extension $F / K$ in the following lemma.

LEMma 5. Let $F / K$ be an inseparable but not purely inseparable algebraic field extension such that $F=S \otimes_{K} J$ where $J / K$ has a modular base. Then there exists an intermediate field of $F / K$ over which $F$ is modular and which is an exceptional and reliable extension of $K$ if and only if $\left(K^{p^{-1}} \cap J\right) / K$ is not simple. 
Proof. If such an intermediate field exists, then $\left(K^{p^{-1}} \cap J\right) / K$ is not simple by [7, Theorem 4, p. 46]. Conversely, suppose $\left(K^{p^{-1}} \cap J\right) / K$ is not simple. Then $J / K$ is not simple. Let $M$ be a modular base of $J / K$ and let $u, v$ be distinct elements of $M$. Let $n, t$ denote the exponents of $u, v$ over $K$, respectively. Suppose $n \geqslant t$. Let $s \in S-K$. Set $E=K\left(s u p^{n-t}+v\right)$. Now $s \in E$, $E / K(s)$ is simple, and $K(s)$ is the maximal separable intermediate field of $E / K$. Either $E \cap J \supset K$ or $E \cap J=K$. If $E \cap J \supset K$, then $K\left(s^{\left.p^{t-1} u p^{n-1}+u p^{t-1}\right) / K}\right.$ splits as can be seen by a simple degree argument. However this is impossible since, by [7, Theorem 4, p. 47], $K\left(s^{p^{t-1}} u p^{n-1}+v p^{t-1}\right) / K$ is exceptional. Thus $E \cap J=K$ so $E / K$ is exceptional. A similar argument shows that $E$ does not split nontrivially over any intermediate field of $K(s) / K$. Hence by the comments preceding [7, Theorem 1, p. 44], $E$ does not split nontrivially over any intermediate field. Thus $E / K$ is reliable by [7, Theorem 1, p. 44]. Since $v \in E(u), M-\{v\}$ is a modular base of $J E / E$. Since also $F=S E \otimes_{E} J E, F / E$ is modular. Q.E.D.

THEOREM 3. Suppose $K$ is a Galois subfield of $L$. Then the following conditions are equivalent.

(1) Every Galois intermediate field of $L / K$ splits over $K$.

(2) Every intermediate field of $L / K$ splits over $K$.

(3) Every intermediate field of $L / K$ is Galois and splits over $K$.

(4) Every intermediate field of $L / K$ is Galois, splits over $K$, and is modular over $K$.

(5) $L / S$ is simple where $S$ is the maximal separable intermediate field of $L / K$.

Proof. That (4) implies (3), (3) implies (2), and (2) implies (1) is immediate.

(5) implies (4): Let $F$ be an intermediate field of $L / K$. Since $K$ is a Galois subfield of $L, L / K$ splits. Thus $L / F$ splits, i.e., $L=S F \otimes_{F} J F$ where $J$ is the maximal purely inseparable intermediate field of $L / K$. Since $L / S$ is simple, $J F / F$ is simple whence modular. Since also $S F / F$ is normal, $F$ is Galois. If $F$ is an intermediate field of $S / K$ or $J / K$, then $F / K$ splits trivially. Suppose $F / K$ is inseparable but not purely inseparable. Since $L / S$ is simple, $J / K$ is simple. Hence $\left(K^{p-1} \cap J\right) / K$ is simple. Thus $F / K$ is not exceptional by [3, Theorem 6, p. 546] Let $J^{\prime}$ be the maximal purely inseparable intermediate field of $F / K$. Either $F / K$ splits or $F / J^{\prime}$ is exceptional. However $F / J^{\prime}$ is not exceptional or else $L / J^{\prime}$ contains exceptional extensions of $J^{\prime}$ which is impossible since $\left(J^{\prime} p^{-1} \cap J\right) / J^{\prime}$ is simple. Thus $F / K$ splits. Since $L / S$ is simple, $J^{\prime} / K$ is simple whence $F / K$ is modular.

(1) implies (5): Since $L / K$ splits, $L$ splits over every intermediate field. Thus any intermediate field over which $L$ is modular is Galois. Thus by (1) every intermediate field over which $L$ is modular splits over $K$. Hence $\left(K p^{-1} \cap J\right) / K$ is 
simple by Lemma 5. Since $K$ is Galois, $J / K$ is modular. Thus $J / K$ whence $L / S$ is simple. Q.E.D.

Corollary. Suppose $G$ is a Galois subgroup of $A$. Then $L / S$ is simple where $S$ is the maximal separable intermediate field of $L / L^{G}$ if and only if for every subgroup $H$ of $G$ which is Galois, $H_{0}$ is $G_{L}$-invariant.

Proof. Suppose $L / S$ is simple. Then $L^{H} / L^{G}$ splits by Theorem 3. Hence by [4, Theorem 4.2, p. 199], $H_{0}$ is $G_{L}$-invariant. Conversely, suppose $H_{0}$ is $G_{L}$-invariant for every subgroup of $G$ which is Galois. Then by [4, Theorem 4.2, p. 199], $L^{H} / L^{G}$ splits for every subgroup $H$ of $G$ which is Galois. Let $F$ be any Galois intermediate field of $L / L^{G}$. Then $A^{F}$ is a Galois subgroup of $G$ and $L^{A} F$ $=F$. Hence $F / K$ splits. Thus $L / S$ is simple by Theorem 3. Q.E.D.

4. Galois subfields. Let $J / K$ be a purely inseparable field extension. If $J / K$ has a modular base, then $J / K$ is modular [8, Proposition 1.23, p. 16] .

Proposition 3. Suppose $J / K$ is a purely inseparable field extension. Then every intermediate field of $J / K$ has a modular base over $K$ if and only if $J^{p} \subseteq K$, or $J / K$ is simple, or $J / K$ has a modular base consisting of two elements.

Proof. Suppose every intermediate field of $J / K$ has a modular base over $K$. Suppose $J / K$ does not have exponent 1 or $J / K$ is not simple. Then if $M$ is a modular base of $J / K, M$ consists of at least two elements one of which has exponent $\geqslant 2$ over $K$, say $m_{1}$. Suppose $M$ has two other elements, say $m_{2}, m_{3}$, with exponents $n, t$ over $K$, respectively. Then $K\left(m_{1}, m_{1} m p^{n-1}+m_{3}^{p^{t-1}}\right)$ is an intermediate field of $J / K$ which does not have a modular base over $K$, a contradiction. Thus $M$ consists of exactly two elements. The converse follows by $[8$, Proposition 2.5, p. 76]. Q.E.D.

LEMMA 6. Suppose $J / K$ is a purely inseparable field extension.

(1) If $\left[K: K^{p}\right]=p^{2}$, then $J / K$ is modular.

(2) If $J / K$ has a modular base and $J / F$ is modular for every intermediate field $F$, then $J / K$ has a bounded exponent.

Proof. (1) Clearly $K^{p^{-i}} / K$ has a modular base consisting of two elements, $i=1,2, \ldots$ By Proposition $3,\left(K p^{-i} \cap J\right) / K$ has a modular base, $i=1,2, \ldots$. By [6, Lemma 2, p. 336] $J=\bigcup_{i=1}^{\infty}\left(K^{p^{-i}} \cap J\right)$ is modular over $K$.

(2) Suppose $J / K$ does not have bounded exponent. Let $M$ be a modular base of $J / K$. There exists $m_{i} \in M$ such that $m_{i}$ has exponent $e_{i}$ over $K$ with $e_{i}<$ $e_{i+1}, i=0,1, \ldots$ Set $F=K\left(M^{\prime}\right)\left(m_{1}^{p^{2}}, m_{2}^{p^{2}},-m_{1}^{p} m_{0}+m_{2}^{p}\right)$ where $M^{\prime}=$ $M-\left\{m_{1}, m_{2}\right\}$. Then $J=F\left(m_{1}, m_{2}\right)$. Now $e_{2}>e_{1} \geqslant 2$ so $m_{1}$ and $m_{2}$ have exponent 2 over $F$ while $m_{2}$ has exponent 1 over $F\left(m_{1}\right)$. Since $m_{0}, m_{1}^{p^{2}}$, 
$-m_{1}^{p} m_{0}+m_{2}^{p}$ are $p$-independent in $F$, it follows that $J / F$ is not modular ([5, Exercise 6, p. 196] or [8, Example 1.59 , p. 55]), a contradiction. Thus $J / K$ has bounded exponent. Q.E.D.

THEOREM 4. Suppose $J / K$ is a purely inseparable field extension. Then $J / F$ has a modular base for every intermediate field $F$ if and only if (1) $J^{p} \subseteq K$, or (2) $J / K$ is simple, or (3) $\left[K: K^{p}\right] \leqslant p^{2}$ and $J / K$ has bounded exponent, or (4) $J / K$ has a modular base in which no more than one element has exponent $\geqslant 2$ over $K$.

Proof. Suppose $J / F$ has a modular base for every intermediate field $F$. Suppose further that (1), (2), and (3) do not hold. By (2) of Lemma $6, J / K$ has a bounded exponent. Since (3) does not hold, $\left[K: K^{p}\right]>p^{2}$. Let $M$ be a modular base of $J / K$. Since (1) does not hold, there exists $m_{1} \in M$ such that $m_{1}$ has exponent $\geqslant 2$ over $K$. Since ( 2 ) does not hold, $M$ has at least two elements. Suppose there exists $m_{2} \in M$ such that $m_{2} \neq m_{1}$ and $m_{2}$ has exponent $\geqslant 2$ over $K$. Since $\left[K: K^{p}\right]>p^{2}$ and $M$ is a modular base of $J / K$, there exists $m_{0} \in J$ such that $m_{0}, m_{1}^{p^{2}},-m_{1}^{p_{1}} m_{0}+m_{2}^{p}$ are $p$-independent in $F$ where $F=$ $K\left(M^{\prime}\right)\left(m_{1}^{p^{2}}, m_{2}^{p^{2}},-m_{1}^{p} m_{0}+m_{2}^{p}\right)$ and $M^{\prime}=M-\left\{m_{1}, m_{2}\right\}$. Now as in the proof of (2) of Lemma $6, J / F$ is not modular, a contradiction. Thus $m_{1}$ is the only element of $M$ with exponent $\geqslant 2$ over $K$.

The converse is immediate if either (1) or (2) holds. Suppose (3) holds. Let $F$ be an intermediate field of $J / K$. Now $\left[F: F^{p}\right] \leqslant p^{2}$. Thus $J / F$ is modular by (1) of Lemma 6 . Since also $J / F$ has bounded exponent, $J / F$ has a modular base. Suppose (4) holds. Let $F$ be an intermediate field of $J / K$. If $J^{p} \subseteq F$, then $J / F$ has a modular base. Suppose $J^{p} \nsubseteq K$. Let $M$ be a modular base of $J / K$. Then $M$ contains a relative $p$-base of $J / F$, say $M^{\prime}$. Since $M^{\prime}$ is a minimal generating set of $J / F$ and $J^{p} \notin F, M^{\prime}$ contains an element $m$ of exponent $\geqslant 2$ over $F$. By (4) and the fact that $M^{\prime} \subseteq M,\left(M^{\prime}-\{m\}\right)^{p} \subseteq K \subseteq F$. Thus $M^{\prime}$ is a modular base of $J / F$. Q.E.D.

Condition (4) of Theorem 4 is equivalent to the existence of a finite iterative higher derivation on $J$ with a field of constants $K$ [10, Theorem 2, p. 439].

Corollary. Suppose $J / K$ is a purely inseparable field extension. Then $J / F$ has a modular base and $F / K$ has a modular base for every intermediate field $F$ if and only if (1) $J^{p} \subseteq K$, or (2) $J / K$ is simple, or (3) $\left[K: K^{p}\right] \leqslant p^{2}$ and $J / K$ has bounded exponent, or (4) $J / K$ has a modular base consisting of two elements with no more than one element having exponent $\geqslant 2$ over $K$.

Proof. Immediate from Proposition 3 and Theorem 4. Q.E.D.

Proposition 4. Suppose $K$ is a Galois subfield of $L$. Then every intermediate field of $L / K$ is Galois if and only if $L$ is modular over every intermediate 
field of $L / S$ where $S$ is the maximal separable intermediate field of $L / K$.

Proof. Suppose every intermediate field of $L / K$ is Galois. Let $F$ be an intermediate field of $L / S$. Then $F$ is Galois so $L / F$ is modular. Conversely, suppose $L$ is modular over every intermediate field of $L / S$. Let $F$ be an intermediate field of $L / K$. Since $L / K$ splits, $L / F$ splits, i.e., $L=S F \otimes_{F} J F$ where $J$ is the maximal purely inseparable intermediate field of $L / K$. Now $L / S F$ is modular so $F J / F$ is modular by the equivalence of (2) and (3) of Lemma 4 . Since $S / K$ is normal, $S F / F$ is normal. Thus $F$ is Galois. Q.E.D.

\section{REFERENCES}

1. R. Davis, A Galois theory for a class of purely inseparable field extensions (unpublished notes).

2. J. Deveney, An intermediate theory for a purely inseparable Galois theory, Trans. Amer. Math. Soc. 198 (1974), 287-295.

3. R. W. Gilmer, Jr. and W. Heinzer, On the existence of exceptional field extensions, Bull. Amer. Math. Soc. 74 (1968), 545-547. MR 36 \#5107.

4. N. Heerema, A Galois theory for inseparable field extensions, Trans. Amer. Math. Soc. 154 (1971), 193-200. MR 42 \#4527.

5. N. Jacobson, Lectures in abstract algebra. Vol. III: Theory of fields and Galois theory, Van Nostrand, Princeton, N. J., 1964. MR 30 \#3087.

6. L. A. Kime, Purely inseparable, modular extensions of unbounded exponent, Trans. Amer. Math. Soc. 176 (1973), 335-349. MR 47 \#192.

7. J. N. Mordeson and W. W. Shoultz, p-bases of inseparable field extensions, Arch. Math. (Basel) 24 (1973), 44-49. MR 47 \#6665.

8. J. N. Mordeson and B. Vinograde, Structure of arbitrary purely inseparable extension fields, Lecture Notes in Math., vol. 173, Springer-Verlag, Berlin and New York, 1970. MR 43 \#1952.

9. M. E. Sweedler, Structure of inseparable extensions, Ann. of Math. (2) 87 (1968), 401-410; correction, ibid. (2) 89 (1969), 206-207. MR 36 \#6391; 38 \#451.

10. M. Weisfeld, Purely inseparable extensions and higher derivations, Trans. Amer. Math. Soc. 116 (1965), 435-449. MR 33 \#122.

DEPARTMENT OF MATHEMATICS, CREIGHTON UNIVERSITY, OMAHA, NEBRASKA 68131 